\title{
Developing an Automated System for Shoe Sole Halogenation
}

\author{
João Sepúlveda \\ Department of Industrial Electronics \\ University of Minho \\ 4800 Guimarães, Portugal \\ João Monteiro \\ Department of Industrial Electronics \\ University of Minho \\ 4800 Guimarães, Portugal
}

\author{
Carlos Couto \\ Department of Industrial Electronics \\ University of Minho \\ 4800 Guimarães, Portugal
}

\begin{abstract}
This paper describes a project developed in the Department of Industrial Electronics for a shoe wear manufacturer. It envisaged the study and automation of a human operated position of a shoe production line.

A trade of investment and operational costs, technical feasibility and environmental benefits pointed to the selection of the shoe's sole halogenation position. This operation, manually performed, is done before glue application. Its purpose is the preparation of the shoe sole to receive, in good conditions, the glue. The chemical material used is toxic and harmful to operators. The developed system integrates a manipulator and an artificial vision system.

The results that emerged from the experimental tests demonstrated that the proposed automated system works as desired. The system exhibits the additional advantage of performing the halogenation procedure in almost half of the time required by a human operator.

The image processing algorithms included in the system software are fast enough to enable image acquisition and processing as well as the transmission of the path to the robot controller, on line.
\end{abstract}

\section{INTRODUCTION}

The aim of this paper is to describe a robotic system for shoe sole halogenation. Shoe soles need a good cleaning before they can be glued to the shoe body. Often, the cleaning procedure is performed through a chemical process, comprising a vigorous friction with a short brush with a halogenating liquid, around the sole border. This liquid is very harmful for the worker.

Using a robot to do this kind of task is very desirable, since it can work on a closed cell and production rates can be increased. However, there is a major problem concerning the variety of shoe soles: many sizes, right and left foot, and many kinds of models [4].

\section{THE DEVELOPED SYSTEM}

The solution to this problem comprises the integration of an artificial vision system associated with the robot. The vision system consists in a small surveillance CCD camera, a low cost video acquisition card - the Video Blaster, and a 486DX33 personal computer with 8 Mega Bytes of RAM. The camera is oriented perpendicularly to the sole, the video card acquires the image in 256 levels of grey, then the image is converted to black \& white, the border is computed and then the dominant points are determined. After that, the robot end effector (i.e. the brush) moves along these points, applying the halogenating liquid.

The used robot is the MITSUBISHI RV-M2, a five-degree of freedom manipulator with DC servomotors plus a grip. It is possible to use an electric or a pneumatic grip, but the electric is more convenient because it is easier to use, since the robot controller provides its power. The pressure and velocity are easily adjusted by software and last, it has a wider opening [5], very useful to this application.

The robot controller is the DUM2-V5. It has four buttons on the front panel: emergency stop, start button, stop button and reset button. On the right side there is a door which accesses one of the controller's cards where can be inserted an EPROM with the robot's program, there is a backup battery which preserves data and the program in the RAM even if the unit is turned off. There are also several LED's to indicate error messages and some dip switches to configure the system. In the backside there is the main power switch, a grip type selector, a connector for a remote emergency stop and four removable cards [5].

The first card is used to communicate with a personal computer through a serial port RS-232C (bi-directional) or through a CENTRONICS parallel port (unidirectional).

The second is used to connect a teaching box and provides sixteen digital inputs and sixteen digital outputs to connect to the peripherals. The teaching box is not intended to programme the robot, it is only used to move the joints with articulated or linear interpolation, open or close the grip, insert or delete positions and execute programs step by step [5].

Another card is responsible for feeding the joint encoders and receives their signals. It also has a connector for a sixth robot joint.

The last provides the power to the five manipulator's DC motors.

The instruction set may be divided in six groups: position/motion control instructions, program control instructions, hand control instructions, I/O control instructions, RS-232C read instructions and miscellaneous instructions [5].

The robot's program can be memorised in the controller's EPROM or RAM or in the computer [5], which is more suitable to this application. Since the points defining the robot trajectories vary from sole to sole and a computer was needed to determine each sole's dominant points.

Fig. 1 shows a general view of the system. 


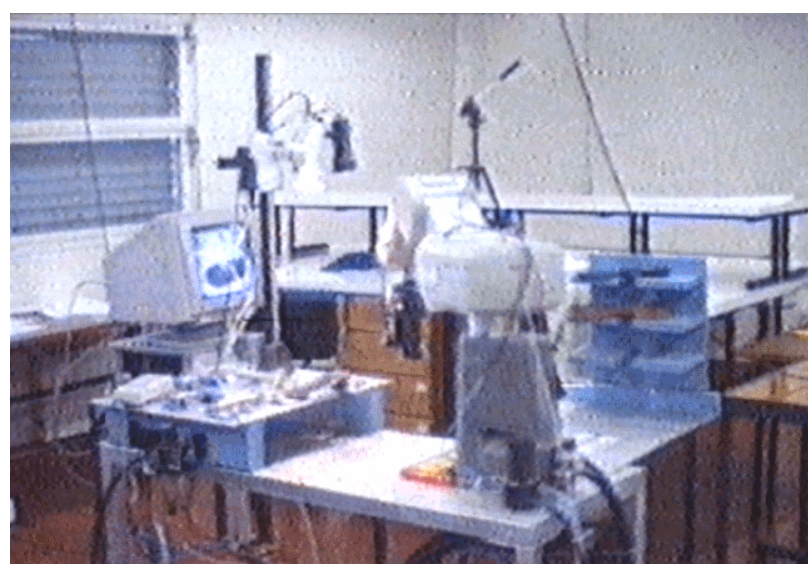

Fig. 1. General view of the system for automated shoe sole halogenation

The robot is quite heavy and very fast, having the tendency to move the base, so it was necessary to build a metal table to support the robot and all the components. It is important to note that the relative positions of the components can't be altered.

Over the support table there was introduced a "working table" consisting in a small metal table where the shoe soles are processed. There are also two pneumatic cylinders controlled by electromagnetic valves equipped with specially designed clamps, one rigidly fixed to the cylinder axle and the other with pivoted joint, that can cope with different kinds and sizes of soles. Double effect cylinders and spring cylinders were tried, but the results were identical.

In Fig. 2 is shown the working table with a sole and pneumatic cylinders for shoe sole holding. Electromagnetic valves, also shown in Fig. 2 control the cylinders. It was also necessary to introduce a pressure regulator, since without it, the cylinders either wouldn't work due to lack of pressure or smashed the sole if the pressure was too high. The working table was painted in matt white in order to provide high contrast with the sole to ease image processing.

The robot's end effector was also changed: larger fingers and a rotary brush were added in order to manipulate the sole and apply the liquid. In Fig. 3 the end effector is shown.

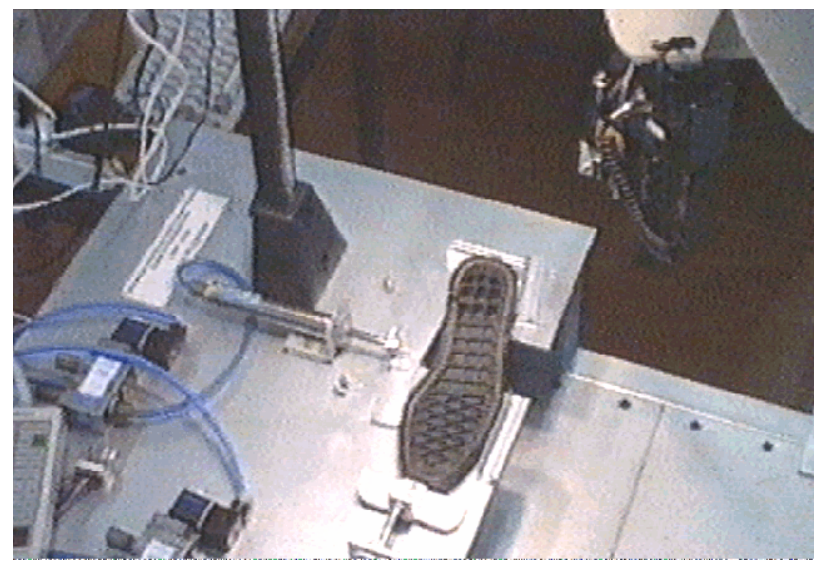

Fig. 2. Working table with a sole and pneumatic cylinders

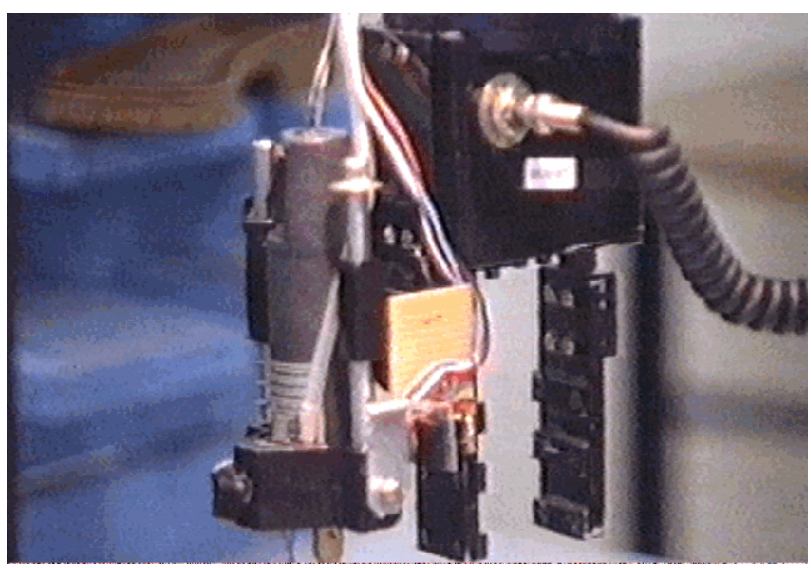

Fig. 3. Robot's end effector

The rotary brush is DC motor driven implemented with a PCB power drill equipped with a short brush and mounted on the robot's grip. By rotating the wrist, the robot changes from grabbing to brushing position and vice-versa. The $12 \mathrm{~V}$ DC motor is powered by pulse width modulation from $200 \%$ of its rated voltage, providing low speed and high starting torque.

The halogenating liquid is fed through a tubed needle. The needle from an ink jet printer refill was used, pointed to the brush. A thin flexible pipe connects the needle to an electric pump mounted near the robot base. This pump is an ordinary $12 \mathrm{~V}$ car's petrol pump, powered by the same technique as the rotary brush, i. e. PWM voltage obtained from a $24 \mathrm{~V}$ bus bar.

The electromagnetic valves, the brush and the pump are controlled by the robot.

The rotary brush, the pipe and the needle can be seen in Fig. 3.

It is important to mention that the halogenating liquid is very thin and volatile, so it is not necessary to take any special care about brush or needle cleaning.

The camera is placed in a photo three-degree of freedom support perpendicularly with the table, and so, with the sole. It makes possible to determine only two dimensions of the sole. It is necessary to determine the sole's height.

Some solutions to this problem were proposed:

A second camera would solve the problem, but this would be an expensive solution and the image processing would become more complicated.

A single camera positioned in an oblique orientation was also possible, but the image processing algorithms would be very complicated and take a larger amount of time.

The solution adopted was to have the rotary brush fixed to a spring-loaded support. This arrangement allows the vertical movement of the brush against the spring. Two optical switches detect when the spring is off loaded or fully loaded, i. e. brushing with excessive pressure.

Those switches can also be seen in Fig. 3 and are connected to the robot controller. The idea is to keep one of the switches on and the other off. In Fig. 4 there is a more comprehensive diagram of the brush mounting. 


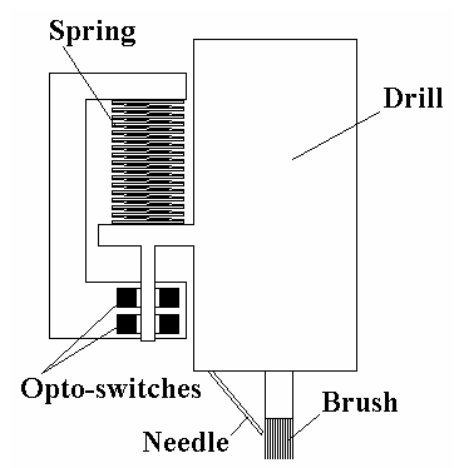

Fig. 4. Brush mounting diagram and opto-switches

The robot controller does not allow interrupts, so the program was altered to periodically verify the state of the inputs coming from the optical switches, while the sole is being brushed, and alters the robot tool length if necessary.

If both switches are off, the brush is too high, so the tool length is decreased, causing the arm to go down, since the robot coordinates are relative to the tip of the "fingers".

If both switches are on, the brush is too low, so the tool length is increased in order to make the arm go up.

If the lower switch is on and the upper is off, then the height and so, the brushing pressure is correct.

It is very important to notice that, as many components were added to the robot, the working volume was reduced, since it is more difficult to avoid collisions and more care should be taken. Also, as some components were added to the robot hand, its capability for lifting objects (shoe soles) is reduced but it is still enough to lift the heaviest sole at maximum speed [3].

The robot controller has inputs, outputs and a parallel port, which is connected to the computer. The computer provides only information on each sole and sets the program flow. The rotating brush, liquid pump, air valves and height sensors are connected to the robot controller by a small interface board that can be seen in Fig. 5 .

The parallel port was preferred because it is much faster than the serial port, although it is only unidirectional, not allowing the robot controller to send messages to the computer (error messages, for example).

The optical switches can be directly connected to the controller inputs, providing information on the sole's height.

As for the rotary brush, the liquid pump and the electromagnetic valves, it is not possible to connect them directly to the robot controller. It was necessary to build a small interface card and use an external power supply that can also be seen in Fig. 5. This interface board is very simple, consisting on four power transistors, three NE555 timers, some resistors and some capacitors. Two potentiometers allow adjusting the speed of the brush and the pressure of the pump.

\section{PROCEDURE SEQUENCE}

The developed program ensures the following procedure sequence:

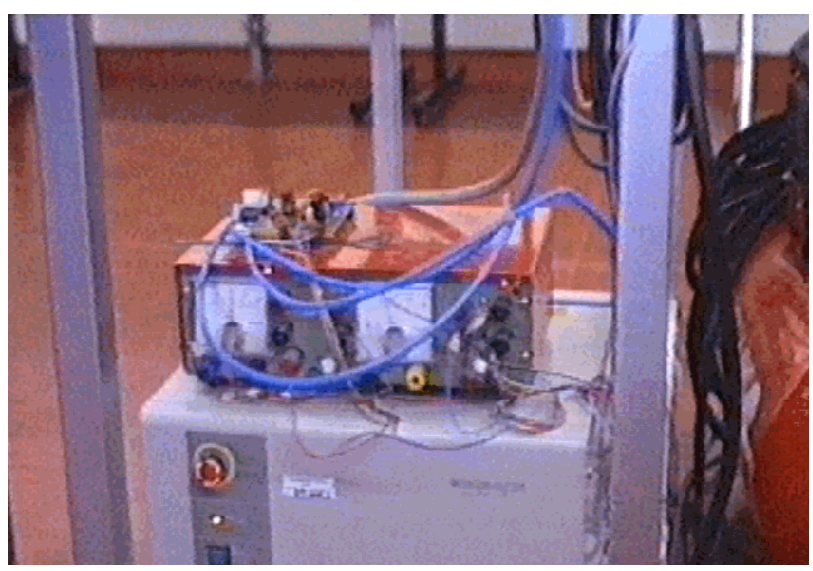

Fig. 5. Robot controller, power supply and interface board

1. The manipulator picks a sole from the shelf and puts it on the table.

2. The controller actuates the electromagnetic valves in order to make the cylinders to hold the sole.

3. The manipulator turns the end effector to the brushing position, by wrist pitch rotation.

4. The image is acquired, after the manipulator stops, since it is necessary that the table does not shake.

5. Through an image processing program, the computer determines the sole's dominant points.

6. The computer sends the points to the robot controller via the parallel port.

7. The manipulator moves to a position above the sole.

8. The pump and the rotating brush are turned on.

9. The manipulator applies halogenating liquid on the sole's border with the brush.

10. The pump and the rotating brush are turned off.

11. The end effector is placed on grabbing position.

12. The manipulator catches the sole and puts it back on the shelf.

The robot only processes the sole while it is sitting on the working table. While the sole is being moved, no "work" is done. So, in order to minimise this time, the robot moves the sole at maximum speed, with top acceleration and using articulated interpolation.

While the sole is being brushed, the velocity and acceleration are reduced to ensure a more perfect work.

\section{THE VISION SYSTEM [2]}

The vision system, a low cost solution, is composed by a personal computer with a general purpose image acquisition board connected to a surveillance black \& white CCD camera and a mixed software package 
developed in $\mathrm{C}++$ for Windows ${ }^{\circledR}$ and assembly language (to directly access the video board's frame buffer).

The software package contains several modules. The most important are: sole image acquisition, image segmentation, contour extraction and robot control.

Apart from these, other modules were developed, such as calibration, and two other tools, useful during the development stage. These were the display (allows the view of images at any point) and information (allows the inspection of numerical data and results, par example, the points sent to the robot).

One solution that was proposed consisted in using the camera only to identify the type and size of the sole and then, load the sole's points from a file. However this method was not implemented because the used image processing algorithms are fast enough to enable the acquisition, image processing and communication of each sole's coordinates to the robot controller, on line. Two techniques were tried [2]:

First, thresholding/binarization, contour extraction, dominant point calculation (450 to 15 ) and inside parallel contour.

Second, Microsoft Windows ${ }^{\circledR}$ based program, performing thresholding/binarization based on histogram analysis, binary erosion, contour extraction and dominant point calculation (variable number of points). The computer and the image-processing program can be seen in Fig. 6.

The computer needs no special requirements, as it is an ordinary and inexpensive $486 \mathrm{DX}$ at $33 \mathrm{Mhz}$ with $8 \mathrm{Mega}$ bytes of RAM equipped with a Video Blaster card.

The image processing turned also to be much easier and faster due to the painting of the working table in matt white, providing high contrast with the sole, and due to the installation of a convenient lighting system that eliminates the shadows around the soles.

\section{CONCLUSIONS}

The results that emerged from the experimental tests that have been done, demonstrated that the proposed automated system works as desired. The system exhibits the advantage of performing the halogenation procedure in almost half of the time required by a human operator.

Finally, this system enables not only a better productivity, but also a considerable improvement in working environmental conditions.

\section{FUTURE ENHANCEMENTS}

The system can be enhanced if the brush mounting has a more convenient design, as sometimes, if not properly lubricated, the moving base stalls.

The needle that injects the liquid would be better placed inside the brush and not pointing to it.

It would be also preferable to put a reducing gear between the brush and the DC motor, to reduce speed even more and increase the torque.

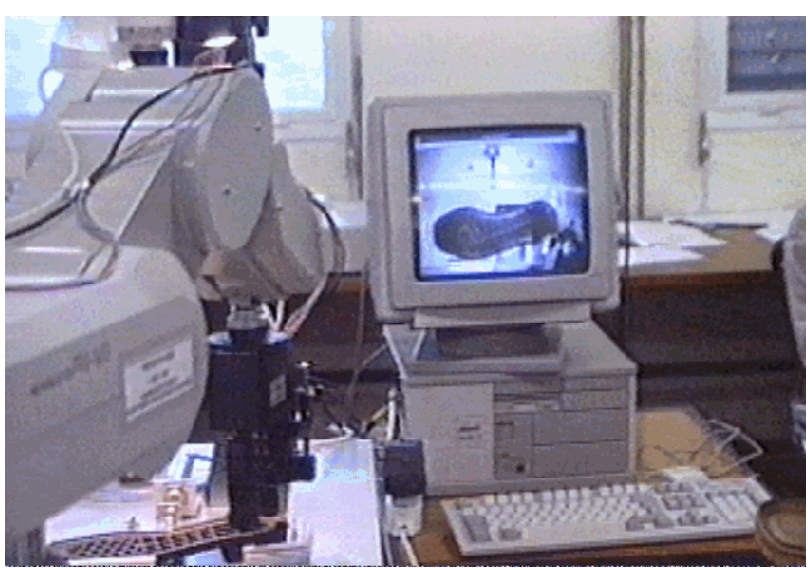

Fig. 6. Computer with Microsoft Windows ${ }^{\circledR}$ based image-processing program

The use of a robot controller allowing interrupts and interrupt service subroutines would result in a more precise control on the sole's height and a faster correction in case of error. Alternatively a PC equipped with a proper interfacing board to emulate the robot controller will also be considered.

Some contacts were made to find an industrial partner to derive the industrial version from this conceptual prototype.

\section{ACKNOWLEDGMENT}

The authors gratefully acknowledge the contributions of Estela Bicho and Júlio Martins.

\section{REFERENCES}

[1] Carlos Couto, João Monteiro, Fátima Santos and Sepúlveda Freitas, "An Automated System for Shoe Sole Halogenation" Communication presented in the A.M.S.E. Conference, Egypt: 1992.

[2] João Monteiro, Adriano Tavares, Carlos Couto, "A Vision System to Derive The Optimum Path in a Robot Based Shoe Halogenation Machine" Proceedings of the $2^{\text {nd }}$ Japan-France Congress on Mechatronics, Takamatsu, Kagawa, Japan: November 1-3, 1994, Volume 1 pp. 79-82.

[3] Rex Miller, Fundamentals of Industrial Robots and Robotics, PWS-KENT Publishing Company: 1988.

[4] Richard K. Miller, Industrial Robot Handbook, Competitive manufacturing series: 1989, pp. 617-620.

[5] MITSUBISHI, INDUSTRIAL MICRO-ROBOT SYSTEM MODEL MOVEMASTER RV-M2 Instruction Manual, pp. 1.16-1.19, pp. 1.13-1.15, p. 1.20 , p. 3.1.

[6] Richard D. Klafter, Thomas A. Chmielewski and Michael Negin, Robotic Engineering: An Integrated Approach, Prentice Hall: 1989. 